\title{
Young measure approach to characterization of behaviour of integral functionals on weakly convergent sequences by means of their integrands
}

by

\author{
M. SYCHEV* \\ Sobolev Institute of Mathematics, Novosibirsk 630090, Russia. \\ E-mail: masychev@math.msc.ru
}

\begin{abstract}
It is known that sequential weak lower semicontinuity and weak-strong convergence (in the scalar case) properties of integral functionals may be characterized by means of their integrands. In this paper we introduce a Young measure approach obtaining both these results and the characterization for the second property in the vector-valued case. We discuss also motivations for the definition of strict quasiconvexity, and point out that the characterization of the classes of functionals having weak-strong convergence property everywhere is not a trivial problem in the general case.
\end{abstract}

C 1998 L'Association Publications de l'Institut Henri Poincaré. Published by Elsevier B.V. All rights reserved

Key words: Integral functionals, gradient Young measures, stability.

RÉSUMÉ. - On sait que les propriétés de semicontinuité séquentielle faible et de convergence faible-forte (dans le cas scalaire) pour les intégrales fonctionnelles peuvent être caractérisées au moyen de leurs intégrants. Dans ce papier nous introduisons une approche pour la mesure de Young et nous obtenons ces résultats ainsi que la caractérisation de la seconde propriété dans le cas à valeur vectorielle. Nous discutons également les motivations pour définir la quasiconvexité stricte, et remarquons que la caractérisation des classes de fonctionnelles ayant partout la propriété de convergence faible-forte n'est pas un problème trivial dans le cas général. (C) 1998 L'Association Publications de l'Institut Henri Poincaré. Published by Elsevier B.V. All rights reserved

* Supported by DAAD and ICTP during author's visit in 1995 in Freiburg University and International Centre for Theoretical Physics respectively.

Annales de l'Institut Henri Poincaré - Analyse non linéaire - 0294-1449 Vol. 15/98/06/

(C) 1998 L'Association Publications de l'Institut Henri Poincaré. Published by Elsevier B.V. All rights reserved 


\section{INTRODUCTION}

In this paper we will consider integral functionals

$$
I(u)=\int_{\Omega} L(x, u(x), \nabla u(x)) d x
$$

where $L(x, u, v): R^{n} \times R^{m} \times R^{n m} \rightarrow R$ is a Caratheodory integrand, $u \in W^{1,1}\left(\Omega ; R^{m}\right)$. We define $I(u)$ as the integral in the right-hand side if the composition of $L$ and $u$ lies in $L^{1}$, and let $I(u)=\infty$ if only the negative part of this composition is integrable. We will suppose that $\Omega \subset R^{n}$ is a bounded open set with the boundary having zero Lebesgue measure unless otherwise stated. In this case $W^{1, p}\left(\Omega ; R^{m}\right)$ is the space consisting of measurable functions with finite norm $\|u\|_{W^{1, p}\left(\Omega ; R^{m}\right)}:=\|u\|_{L^{p}\left(\Omega ; R^{m}\right)}+\|\nabla u\|_{L^{p}\left(\Omega ; R^{n m}\right)}$, $W_{0}^{1, p}\left(\Omega ; R^{m}\right)$ is the closure of $C_{0}^{\infty}\left(\Omega ; R^{m}\right)$ in $W^{1, p}\left(\Omega ; R^{m}\right)$.

The main purpose of the paper is to obtain a characterization by means of integrands of two basic properties of these functionals. The two properties are: sequential weak lower semicontinuity (swlsc) (lim inf $\lim _{k \rightarrow \infty} I\left(u_{k}\right) \geq$ $I\left(u_{0}\right)$ for $u_{k} \rightarrow u_{0}$ in $\left.W^{1, p}\left(\Omega ; R^{m}\right)\right)$ and so-called weak-strong convergence property (the convergences $I\left(u_{k}\right) \rightarrow I\left(u_{0}\right)<\infty$ and $u_{k} \rightarrow u_{0}$ in $W^{1, p}\left(\Omega ; R^{m}\right)$ imply strong convergence of $u_{k}$ to $u_{0}$ in $\left.W^{1,1}\left(\Omega ; R^{m}\right)\right)$. Here and further we will denote weak and strong convergence by $\rightarrow$ and $\rightarrow$ respectively.

When the first property is basic for establishing existence results in a minimization problem (cf. [4], [11], [12]) the second one is relevant for investigation of stability of solutions, convergence in numerical schemes, etc.

The basic conception for definitions and results in this paper is the observation that an integral functional $I(u)$ admits one of the discussed properties at a function $u_{0}$ if and only if for a.e. $y \in \Omega$ the functional with the integrand $L\left(y, u_{0}(y), v\right)$ (y is fixed and determines the integrand) admits the same property at the linear function with gradient $\nabla u_{0}(y)$.

Following this way we unify previous background on these problems both in sense of conceptions and proofs, introduce definition of strict quasiconvexity which turns out to be responsible for the weak-strong convergence property, and partially characterize the class of functionals having this property everywhere. The basic tool in this work will be results from gradient Young measure theory that is motivated by recent contributions of Kinderlehrer \& Pedregal [18]-[20] in this area.

It is well known that the following definition of Morrey [27] is relevant for lower semicontinuity results. 
DeFinition 1.1. - A function $L(v): R^{n m} \rightarrow R$ is quasiconvex at $v_{0} \in R^{n m}$ if

$$
J(u)=\int_{\Omega} L\left(v_{0}+\nabla \varphi(x)\right) d x \geq L\left(v_{0}\right) \text { meas } \Omega
$$

for all $\varphi \in C_{0}^{\infty}\left(\Omega ; R^{m}\right)$ (here $\left.u(x)=v_{0} x+\varphi(x), x \in \Omega\right)$.

A function is called quasiconvex if it is quasiconvex everywhere.

Here and further for a $v_{0} \in R^{n m}$ we suppose that $x \rightarrow v_{0} x$ is a function $R^{n} \rightarrow R^{m}$ given by action of $v_{0}$ as an element of $M^{m \times n}$, which is the space of all $m \times n$ matrices with real components, on $x \in R^{n}$.

Further we will also use the notation $J(u)$ for integral functionals with integrands $L=L(\nabla u)$ depending on $\nabla u$ only for to distinguish this case from the general one, for which the notation $I(u)$ has been reserved.

It is well known that quasiconvexity at a fixed point does not depend on a choice of $\Omega$ [6]. Moreover, arguments of Proposition 2.3 from [6] let prove also.

Proposition 1.2. - A continuous integrand $L(v)$ is quasiconvex at $v_{0}$ if and only if the corresponding functional $J(u)$ is sequentially weakly* lower semicontinuous in $W^{1, \infty}\left(\Omega ; R^{m}\right)$ at the function $u_{0}$ with $u_{0}(x)=v_{0} x$.

The following result of Acerbi \& Fusco [1] is a characterization of the class of functionals having the sequential weak lower semicontinuity property everywhere.

Recall that

$L(x, u, v): R^{n} \times R^{m} \times R^{n m} \rightarrow R$ is a Caratheodory integrand if and only if for any $\varepsilon>0$ there exists a compact subset $\Omega_{\epsilon}$ of $\Omega$ such that meas $\left(\Omega \backslash \Omega_{\varepsilon}\right) \leq \varepsilon$, and the restriction of $L$ to $\Omega_{\varepsilon} \times R^{m} \times R^{\text {nm }}$ is continuous .

THEOREM 1.3. - Let $L(x, u, v)$ be a Caratheodory integrand such that $0 \leq L(x, u, v) \leq A|v|^{p}+B(A, B>0)$. Then the functional $I(u)$ is swlsc in $W^{1, p}\left(\Omega ; R^{m}\right)$ if and only if for a.e. $x \in \Omega$ and all $u \in R^{m} L(x, u, v)$ is a quasiconvex function of $v$.

In the case $p=\infty$ it is enough to demand boundedness of $L$ on compact sets instead of growth conditions at infinity [27].

In the scalar case $\min \{m, n\}=1$ both sequential weak lower semicontinuity and weak-strong convergence properties may be characterized even by means of pointwise properties of integrands.

DEFINITION 1.4. - A continuous function $L(v): R^{l} \rightarrow R$ is convex (strictly convex) at a point $v_{0} \in R^{l}$ if $\sum_{i} c_{i} L\left(v_{i}\right) \geq L\left(v_{0}\right)\left(>L\left(v_{0}\right)\right.$ ) for any $v_{i} \neq v_{0}, c_{i} \geq 0(i=1, \ldots, q)$ such that $\sum_{i} c_{i} v_{i}=v_{0}, \sum_{i} c_{i}=1$. 
Convexity of a function $L$ at a point $v_{0}$ is equivalent to nonemptiness of the subgradient at this point. Strict convexity at $v_{0}$ holds if and only if for a $f \in \partial L\left(v_{0}\right)$ the convex hull of the set $\left\{v \in R^{l}, v \neq v_{0}: L(v)-L\left(v_{0}\right)-<\right.$ $\left.f, v-v_{0}>=0\right\}$ does not contain $v_{0}$ (as a consequence the same is true for any element of the subgradient). All these results are the contents of $[30, \S 2]$.

In Theorem 1.5 a more general situation will be considered, in which gradients are replaced by arbitrary integrable functions. In this case

$I(u, \xi)=\int_{\Omega} L(x, u(x), \xi(x)) d x, \quad L(x, u, v): \Omega \times R^{m} \times R^{l} \rightarrow R, \Omega \subset R^{n}$.

ThEOREM 1.5. - Let $L(x, u, v)$ be a Caratheodory function, $u_{k} \rightarrow u$ in $L^{1}\left(\Omega ; R^{m}\right), \xi_{k} \rightarrow \xi$ in $L^{1}\left(\Omega ; R^{l}\right)$, and for a.e. $x \in \Omega$ assume that $L(x, u(x), v)$ is convex at $v=\xi(x)$. Let also negative parts of $L\left(x, u_{k}(x), \xi_{k}(x)\right)$ be equi-integrable. Then $\liminf _{k \rightarrow \infty} I\left(u_{k}, \xi_{k}\right) \geq$ $I(u, \xi)$.

If additionally for a.e. $x \in \Omega L(x, u(x), v)$ is strictly convex at $v=\xi(x)$ and $I(u, \xi)<\infty$ then the convergence $I\left(u_{k}, \xi_{k}\right) \rightarrow I(u, \xi)$ implies convergences $\xi_{k} \rightarrow \xi$ and $L\left(x, u_{k}(x), \xi_{k}(x)\right) \rightarrow L(x, u(x), \xi(x))$ in $L^{1}$.

Equi-integrability of a sequence $f_{k}: \Omega \rightarrow R^{l}$ means that for any $\varepsilon>0$ there exists $\delta>0$ such that for any measurable subset $\tilde{\Omega}$ of $\Omega$, for which meas $\tilde{\Omega} \leq \delta$, the inequality $\int_{\tilde{\Omega}}\left|f_{k}\right| \leq \varepsilon$ holds for all $k$.

The first assertion of the theorem has been first proved in [3], the second one in [30] (see also [31] for a slightly different proof, and [32] for a very elementary proof of a weaker assertion). For $L=L(x, v)$ convex in $v$ an optimal condition implying the weak-strong convergence property had been obtained in [33].

In the scalar gradient case $\left(\xi_{k}=\nabla u_{k} \cdot \min \{n, m\}-1, l-m n\right)$ the converse result is valid.

TheOREM 1.6. - Let $L(x, u, v): R^{n} \times R \times R^{n} \rightarrow R$ be a Caratheodory integrand, $u_{0} \in W^{1 . p}(\Omega), p \in\left[1, \infty\left[\right.\right.$. Suppose that $|L(x, u, v)| \leq A|v|^{p}+B$ $A, B>0$.

Then the validity of the inequality $\liminf _{k \rightarrow \infty} I\left(u_{k}\right) \geq I\left(u_{0}\right)$ (the convergence $u_{k} \rightarrow u_{0}$ in $W^{1,1}(\Omega)$ under additional condition $I\left(u_{k}\right) \rightarrow$ $I\left(u_{0}\right)$ ) for all $u_{k} \rightarrow u_{0}$ in $W^{1, p}(\Omega)$ such that $u_{k} \in u_{0}+C_{0}^{\infty}(\Omega)$ and $\left|\nabla u_{k}\right|^{p}$ are equi-integrable implies that for a.e. $x \in \Omega L\left(x, u_{0}(x), v\right)$ is convex at $v=\nabla u_{0}(x)$ (either $L\left(x, u_{0}(x), v\right)$ or $-L\left(x, u_{0}(x), v\right)$ is strictly convex at $v=\nabla u_{0}(x)$ for a.a. $\left.x \in \Omega\right)$. 
In the case $p=\infty$ " $u_{k} \rightarrow u_{0}$ in $W^{1, p}(\Omega)$ " should be replaced by " $u_{k} \rightarrow^{*} u_{0}$ in $W^{1 . \infty}(\Omega)$ ", equi-integrability of $\left|\nabla u_{k}\right|{ }^{p}$ by equi-boundedness of $\left|\nabla u_{k}\right|$. In this case it is enough to require that $|L(x, u, v)| \leq g(x,|u|,|v|)$, where $g$ is nondecreasing in the last two arguments and integrable in $x$ for any fixed $u, v$.

Remark. - Theorem 1.6 was formulated in [30] in a weaker form, but a slight modification of the proof proposed there can be used to obtain an analogous result even in a wider class of integrands: at least for $|L| \leq \theta(v)$, where $\theta(v)$ satisfies $\Delta_{2}$-condition [22] (in this case $u_{0} \in W^{1,1}(\Omega)$ satisfies $\left.\int \theta\left(\nabla u_{0}\right) \leq \infty\right)$.

As for the vector-valued gradient case we can hardly hope to obtain a pointwise characterization for the weak-strong convergence property not having it for swlsc one. Therefore the following definition seems to be reasonable in the context of previous background and in accordance with the general scheme of the results introduced at the beginning.

Derinition 1.7. - A function $L(v): R^{n m} \rightarrow R$ is strictly p-quasiconvex at a point $v_{0} \in R^{n m}$ if it is quasiconvex at this point and for any $c, \varepsilon>0$ there exists $\delta=\delta(c, \varepsilon)>0$ such that the inequalities

$\int_{\Omega} L\left(v_{0}+\nabla \phi(x)\right) d x \leq\left(L\left(v_{0}\right)+\delta\right) \operatorname{meds} \Omega\left(\phi \in C_{0}^{\infty}\left(\Omega: R^{m}\right)\right) .\|\nabla \phi\|_{L^{p}} \leq c$

imply meas $\{x:|\nabla \phi(x)| \geq \varepsilon\} \leq \varepsilon$.

It is worth mentioning that strict $p$-quasiconvexity does not depend on the choice of $\Omega$. This may be established by arguments from Proposition 2.3 from [6].

The above definition just means that for any sequence $\phi_{k} \in C_{0}^{\infty}\left(\Omega ; R^{m}\right)$, which is bounded in $W^{1, p}\left(\Omega ; R^{m}\right)$ and for which

$$
\int_{\Omega} L\left(v_{0}+\nabla \phi_{k}(x)\right) d x \rightarrow L\left(v_{0}\right) \text { meas } \Omega
$$

$\nabla \phi_{k}$ converges to zero in measure. It is easy to see (cf. Proposition 3.4) that for integral functionals of particular form $J(u)$ strict $p$ quasiconvexity of the integrand $L(v)$ at $v_{0}$ is equivalent to the weak-strong convergence property of the functional at the function $v_{0}$. at least for $p>1$ (the same is not proved for the case $p=1$ ).

Strict $p$-quasiconvexity characterizes the weak-strong convergence property in the same fashion as quasiconvexity characterizes swlsc. There exist also possibilities to utilize other similar characterizations (e.g. in Vol. $15, n^{\circ} 6-1998$. 
terms of Young measures), but in this paper we make preference for the above one keeping in mind that swlsc has been also characterized through behaviour of integral functionals of particular form $J(u)$ on linear functions (quasiconvexity). This explains also our preference for terminology in this paper.

Theorem 1.8. - Let $L(x, u, v): R^{\prime \prime} \times R^{m} \times R^{n m} \rightarrow R$ be $a$ Caratheodory integrand, $|L(x, u, v)| \leq A|v|^{p}+B, p \in[1, \infty[. A, B>0$; $u_{0} \in W^{1, p}\left(\Omega ; R^{m}\right)$.

1. Let $u_{k} \rightarrow u_{0}$ in $W^{1, p}\left(\Omega ; R^{m}\right)$ and negative parts of $L\left(x, u_{k}(x), \nabla u_{k}(x)\right)$ be equi-integrable. If for a.e. $x \in \Omega L(x, u(x), v)$ is quasiconvex (strictly p-quasiconvex) at $v=\nabla u_{0}(x)$ then $\liminf \operatorname{in}_{k \rightarrow \infty} I\left(u_{k}\right) \geq I\left(u_{0}\right)$ (the convergence $I\left(u_{k}\right) \rightarrow I\left(u_{0}\right)$ implies convergences $\nabla u_{k_{k}} \rightarrow \nabla u_{0}, L\left(x, u_{k_{k}}(x), \nabla u_{k}(x)\right) \rightarrow L\left(x, u_{0}(x), \nabla u_{0}(x)\right)$ in $L^{1}$ ).

2. Conversely, if for any sequence $u_{k} \in u_{0}+C_{0}^{\infty}\left(\Omega ; R^{m}\right)$ with equiintegrable $\left|\nabla u_{k}\right|^{p}$ the convergence $u_{k} \rightarrow u_{0}$ in $W^{1 . p}\left(\Omega ; R^{\prime n}\right)$ implies the inequality lim inf $f_{k \rightarrow \infty} I\left(u_{k}\right) \geq I\left(u_{0}\right)$ then for a.e. $x \in \Omega L(x, u(x), v)$ is quasiconvex at $v=\nabla u_{0}(x)$. If also $u_{k} \rightarrow u_{0}$ in $W^{1.1}\left(\Omega: R^{m}\right)$ under additional requirements of boundedness below of $L, p>1$, and $I\left(u_{k}\right) \rightarrow I\left(u_{0}\right)$ then for a.e. $x \in \Omega L\left(x, u_{0}(x), v\right)$ is strictly p-quasiconvex at $v=\nabla u_{0}(x)$.

In the case $p=\infty$ " $u_{k} \rightarrow u_{0}$ in $W^{1, p}\left(\Omega ; R^{m}\right)$ " should be replaced by " $u_{k} \rightarrow^{*} u_{0}$ in $W^{1, \infty}\left(\Omega ; R^{\prime \prime}\right)$ ", equi-integrability of $\left|\nabla u_{k}\right|^{p}$ by equiboundedness of $\left|\nabla u_{k}\right|$. In this case it is enough to require that $|L(x, u, v)| \leq$ $g(x,|u|,|\eta|)$, where $g$ is nondecreasing in the last two arguments and integrable in $x$ for any fixed $u, v$.

The previous results on the weak-strong convergence property concern sufficient conditions for this property to hold at a fixed function (everywhere). Such condition had been proposed in [14] and later has been improved in [20], [21]. Morcover, "sufficient" part of our theorem is equivalent to results from [21] in view of Proposition 3.4.

The article will he organized the following way. In $\$ 2$ we include the basic notations and results from Young measure theory. We prove also some auxiliary results which will be used later on.

In $\$ 3$ we give new proofs to Theorems $1.5,1.6$ through results from Young measure theory and prove also Theorem 1.8. We will not consider the case $p=\infty$ in the proofs because this case may be treated by arguments analogous to ones proposed for $p<\infty$. 
In $\$ 4$ we discuss the definition of strict $p$-quasiconvexity utilized in this paper. The term strict $p$-quasiconvexity may seem quite odd. We make preference for it because the property from the definition characterizes weak-strong convergence property in the same way as quasiconvexity characterizes sequential weak lower semicontinuity. Moreover, this property coincides with strict convexity from Definition 1.4 in the scalar case. Anyway we do not insist that our terminology should be of common use.

It may seem natural to suppose that it is enough to demand only strict inequality for $\phi$ not equal identically to zero in the defining inequality for quasiconvexity (this property has been also named as strict quasiconvexity in [24]) or to hope at least that validity of this inequality at every point leads to validity of the weak-strong convergence property. We disprove these hypotheses by counterexamples.

In the same section we consider the problem of characterization of the classes of functionals having sequential weak lower semicontinuity or weakstrong convergence properties everywhere. When it is known (cf. Theorem 1.3) that quasiconvexity of $L(x, u, v)$ in $v$ for a.e. $x \in \Omega$ and all $u$ is precisely a necessary and sufficient condition for the corresponding integral functional to be sequential weak lower semicontinuous everywhere the situation is not analogous in the case of weak-strong convergence property.

It is an easy consequence of results of Alberti [2] and the arguments introduced in the proof of Theorem 1.8 that for integrands of the type $L=L(x, v)$ strict $p$-quasiconvexity (which is strict convexity in the scalar case) in $y$ for a.e. $x \in \Omega$ is both necessary and sufficient condition for the weak-strong convergence property to hold at each function. But in the case of dependence of $L$ on $u$ there exist counterexamples. We will introduce an integrand $L(u, v): R \times R^{2} \rightarrow R$, which is convex, but not strictly, in $v$, in spite of validity of the weak-strong convergence property everywhere. Therefore, the complete characterization of the integrands having the weak-strong convergence property everywhere is quite subtle problem.

\section{BASIC RESULTS IN YOUNG MEASURE THEORY AND SOME AUXILIARY PROPOSITIONS}

Further we will denote by $C_{0}\left(R^{l}\right)$ the set of continuous functions $\Phi$ on $R^{l}$ for which $\lim _{|v| \rightarrow \infty} \Phi(v)=0$. We will denote the set of all probability measures on $R^{l}$ as $M_{1}^{+}$following [21]. In order to distinguish the scalar 
product and the action of a measure on a function we will use notation $<\cdot ; \cdot\rangle$ in the second case.

We will use the following definition of Young measures generated by sequences of functions.

DEFINITION 2.1. - Let $\Omega$ be a measurable bounded subset of $R^{n}$.

A family of probability measures $\left\{\nu_{x}\right\}_{x \in \Omega}$ is said to be the Young measure of the sequence $z_{j}: \Omega \rightarrow R^{l}$ of measurable functions if $\Phi\left(z_{j}\right) \longrightarrow^{*}<\Phi ; \nu_{(\cdot)}>$ in $L^{\infty}$ as $j \rightarrow \infty$ for every $\Phi \in C_{0}\left(R^{l}\right)$.

A Young measure $\left\{\nu_{x}\right\}_{x \in \Omega}$ is named homogeneous if it does not depend on $x$.

In propositions $2.2-2.5$ we will assume that $\Omega$ is a measurable bounded subset of $R^{n}$.

The following result due to Balder [3], Ball [5] improves the original contribution of Young [34], [35].

Theorem 2.2 (Existence theorem). - For any sequence of measurable functions $z_{j}$ for which

$$
\lim _{k \rightarrow \infty} \sup _{j} \operatorname{meas}\left\{x \in \Omega:\left|z_{j}(x)\right| \geq k\right\}=0
$$

there exists a Young measure $\left(\nu_{x}\right)_{x \in \Omega}$ generated (possibly) by a subsequence. If additionally $\operatorname{dist}\left(z_{j}(x), K\right) \rightarrow 0$ for a.e. $x \in \Omega$ and a closed set $K$ then $\operatorname{supp} \nu_{x} \subset K$ for a.e. $x \in \Omega$.

It is well known that

PROPOSITION 2.3. - 1. If $\left\{\nu_{x}\right\}_{x \in \Omega}$ is generated by a sequence $z_{j}^{1}$ and $z_{j}^{2}-z_{j}^{1} \rightarrow 0$ in measure then $z_{j}^{2}$ generates the same Young measure.

2. A sequence $z_{j}$ generates a family of Dirac measures if and only if $z_{j}$ converges in measure.

The next two propositions demonstrate how Young measures may be involved in studying the behaviour of integral functionals on weakly convergent sequences.

PROPOSITION 2.4 ([3], [5], [7]). - Let $z_{k}$ be a sequence with associated Young measure $\left\{\nu_{x}\right\}_{x \in \Omega}$. If $L(x, v)$ is a Caratheodory integrand and the sequence $L\left(x, z_{j}(x)\right)$ is equi-integrable then $L\left(x, z_{j}(x)\right) \rightarrow \int_{R^{\prime}} L(x, v) d \nu_{x}$ in $L^{1}$.

Proposition 2.5. - Let $L(x, v): \Omega \times R^{l} \rightarrow R$ be a Caratheodory integrand and $z_{j}(x): \Omega \rightarrow R^{l}$ be a sequence, which is bounded in $L^{1}$ and such that the negative parts of $L\left(x, z_{j}(x)\right)$ are equi-integrable on $\Omega$. 
If $z_{j}$ generates the Young measure $\left(\nu_{x}\right)_{x \in \Omega}$ then

$$
\liminf _{j \rightarrow \infty} \int_{\Omega} L\left(x, z_{j}(x)\right) d x \geq \int_{\Omega} \int_{R^{\prime}} L(x, v) d \nu_{x} d x .
$$

Moreover, the convergence

$$
\int_{\Omega} L\left(x, z_{j}(x)\right) d x \rightarrow \int_{\Omega} \int_{R^{l}} L(x, v) d \nu_{x} d x<\infty, j \rightarrow \infty
$$

holds if and only if $L\left(x, z_{j}(x)\right)$ are equi-integrable.

The inequality stated above is a result of Balder [3] which is valid also for normal integrands (cf. also [21, p. 17]). The second assertion may be obtained as a consequence of the first one.

Proof. - By Proposition 2.4 equi-integrability of the sequence $L\left(x, z_{j}(x)\right)$ implies that

$$
\int_{\Omega} L\left(x, z_{j}(x)\right) d x \rightarrow \int_{\Omega} \int_{R^{\prime}} L(x, v) d \nu_{x} d x<\infty .
$$

To prove the reverse implication we notice that in view of equiintegrability of negative parts of $L\left(x, z_{j}(x)\right)$

$$
\limsup _{j \rightarrow \infty} \int_{\Omega} L\left(x, z_{j}(x)\right) \geq \int_{\Omega} \int_{R^{l}} L(x, v) d \nu_{x} d x<\infty .
$$

Moreover, lack of equi-integrability of $L\left(x, z_{j}(x)\right)$ leads to the strict inequality. Otherwise, there exists a subsequence $z_{i}$ and $\Omega_{i} \subset \Omega$ such that meas $\Omega_{i} \rightarrow 0$, but $\left\|L^{+}\left(x, z_{i}(x)\right)\right\|_{L^{1}\left(\Omega_{i}\right)} \geq \delta$ for some $\delta>0$. There exists a function $z_{0}(x)$ for which $\int_{\Omega} L\left(x, z_{0}(x)\right) d x<\infty$ (for example $z_{0}(x)=z_{j}(x)$, where $j$ is sufficiently $\operatorname{largc}$ ). If $\tilde{z}_{i}=z_{0}(x)$ for $x \in \Omega_{i}$, $\tilde{z}_{i}=z_{i}(x)$ - otherwise, then because of Proposition 2.3 and the first part of this proposition we obtain

$$
\begin{gathered}
\limsup _{j \rightarrow \infty} \int_{\Omega} L\left(x, z_{j}(x)\right) d x \geq \underset{i \rightarrow \infty}{\limsup } \int_{\Omega} L\left(x, z_{i}(x)\right) d x> \\
\limsup _{i \rightarrow \infty} \int_{\Omega} L\left(x, \tilde{z}_{i}(x)\right) d x \geq \int_{\Omega} \int_{R^{i}} L(x, v) d \nu_{x} d x,
\end{gathered}
$$

that gives a contradiction with the assumption of equality of left and right hand sides.

The proof is completed. 
The following definition of gradient $p$-Young measures $(p \in[1, \infty])$ will be a working tool in this paper. In the case $p=\infty$ we will omit " $p$ " in accordance with the tradition.

DEFINITION 2.6. A family of probability measures $\left(\nu_{x}\right)_{x \in \Omega}$ is a gradient p-Young measure provided there is a sequence $u_{j} \in W^{1, p}\left(\Omega: R^{m}\right)$, which converges weakly in $W^{1, p}\left(\Omega ; R^{m}\right)$, such that $\left|\nabla u_{j}\right|^{p}$ are equi-integrable (\| $\nabla u_{j} \|_{L_{\infty}}$ are equi-bounded in the case $p=\infty$ ) and $\left(\nu_{x}\right)_{x \in \Omega}$ is generated by $\nabla u_{j}$.

The weak limit of $u_{j}$ is called underlying deformation.

The crucial result of Kinderlehrer \& Pedregal [18], [20] is a characterization of gradient $p$-Young measures.

THEOREM 2.7. - Let $\left\{y_{x}\right\}_{x \in \Omega}$ be a family of probability measures such that the function $\left\langle\Phi ; \nu_{(\cdot)}\right\rangle$ is measurable for each $\Phi \in C_{0}\left(R^{n m}\right)$. Then $\left\{\nu_{x}\right\}_{x \in \Omega}$ is a gradient p-Young measure if and only if

(i) there is a $u \in W^{1, p}\left(\Omega ; R^{m}\right)$ such that

$$
\nabla u(x)=\int_{R^{n m}} v d \nu_{x}
$$

in $\Omega$ a.e.

(ii) Jensen's inequality $L(\nabla u(x)) \leq \int_{R^{n m}} L(v) d \nu_{x x}$ holds for a.e. $x \in \Omega$ and any continuous quasiconvex function $L(v)$, which is bounded below and satisfies the inequality

$$
\limsup _{|v| \rightarrow \infty}|L(v)| /|v|^{p}<\infty
$$

(in the case $p=\infty L(v)$ is an arbitrary continuous quasiconvex function).

(iii) the function $\Psi(x)=\int_{R^{n}}|v|^{p} d \nu_{x}$ lies in $L^{1}$ (in the case $p=\infty$ there exists a compact set $C$, such that supp $\left.\left\{\nu_{x}\right\}_{x \in \Omega} \subset C\right)$.

Remark 2.7. - Another important fact from [18], [20] is the observation that in the case $p \in] 1, \infty\left[\right.$ a family of probability measures $\left(\nu_{x}\right)_{x \in \Omega}$, which is generated by gradients of a sequence bounded in $W^{1, p}\left(\Omega, R^{m}\right)$, satisfies all requirements (i)-(iii) of Theorem 2.7. Therefore $\left(\nu_{x}\right)_{x \in \Omega}$ is generated also by a sequence $\nabla u_{k}$ with equi-integrable $\left|\nabla u_{k}\right|^{p}$. In the following we will utilize this result often.

This result may be also obtained as a consequence of stability in the Hodge decomposition [16] (see [21], Theorem 3.10). 
It is worth mentioning that analogous assertion is not valid for the case $p=1$ as it was demonstrated recently by $\mathrm{O}$. Kalamajska [17].

One should mention that here we suppose measurability of the function

$$
x \rightarrow\left\langle\nu_{x} ; \Phi>:=\int_{R^{n m}} \Phi(v) d \nu_{x}(v)\right.
$$

for any $\Phi \in C_{0}\left(R^{n m}\right)$ (later on we utilize notation $\left(\nu_{x}\right)_{x \in \Omega} \in C_{0}\left(R^{n m}\right)^{\prime}$ in this case).

In this case we have automatically that the function

$$
x \rightarrow<\nu_{x} ; L(x, v)>:=\int_{R^{n m}} L(x, v) d \nu_{x}(v)
$$

is measurable for any Caratheodory integrand $L(x, v)$ [3], [21, §3.2]. In the following we will utilize one more result, which is a simple consequence of Theorem 2.7 .

Proposition 2.8. - Let $p \in[1, \infty]$ and let $\left(\nu_{x}\right)_{x \in \Omega}$ be a Young measure satisfying conditions ( $i$ )-(iii) of Theorem 2.7 with this $p$. If $u_{0} \in W^{1, p}\left(\Omega ; R^{m}\right)$ is an underlying deformation for $\left(\nu_{x}\right)_{x \in \Omega}$ then there exists a sequence $u_{j} \in C_{0}^{\infty}\left(\Omega ; R^{m}\right)$ such that $u_{j} \rightarrow 0$ in $W_{0}^{1, p}\left(\Omega ; R^{m}\right),\left|\nabla u_{j}\right|^{p}$ are equiintegrable $\left(\left|\nabla u_{j}\right|\right.$ are equi-bounded in the case $\left.p=\infty\right)$, and $\left(\nabla u_{0}+\nabla u_{j}\right)$ generates $\left(\nu_{x}\right)_{x \in \Omega}$.

Proof. - By Theorem 2.7 there exists a sequence $v_{j}$ such that $\nabla v_{j}$ generates $\left(\nu_{x}\right)_{x \in \Omega}$ as a gradient $p$-Young measure. By standard approximation arguments (cf. [13, Ch. 10]) there exists a sequence $u_{j} \in C_{0}^{\infty}\left(\Omega ; R^{m}\right)$ for which $\left\|u_{j}-v_{j}+u_{0}\right\|_{w_{1, p} \rightarrow 0}$. Automatically $u_{j} \rightarrow 0$ in $W^{1, p}\left(\Omega ; R^{m}\right),\left|\nabla u_{j}\right|^{p}$ are equi-integrable. By Proposition 2.3 $\nabla u_{j}+\nabla u_{0}$ generates $\left(\nu_{x}\right)_{x \in \Omega}$ also.

It completes the proof.

Further we will need also two more remarkable results on gradient $p$-Young measures in accordance with which homogeneous measures with the same local or average action as a gradient $p$-Young measure are also gradient $p$-Young measures (see [18], [20]).

THEOREM 2.9 (The localization principle). - Let $p \in[1, \infty]$ and let $\left(\nu_{x}\right)_{x \in \Omega}$ be a gradient $p$-Young measure. If $u \in W^{1, p}\left(\Omega ; R^{m}\right)$ is an underlying deformation for $\left(\nu_{x}\right)_{x \in \Omega}$ then for a.e. $x \in \Omega$ the measure $\nu_{x}$ is a homogeneous gradient p-Young measure with the center of mass at $\nabla u(x)$.

Vol. 15, $11^{\circ}$ 6-1998 
By definition the averaging operator $A v:\left(\nu_{x}\right)_{x \in \Omega \Omega} \rightarrow A v\left(\nu_{x}\right)_{x \in \Omega}$ gives a probability measure having the same action on every function $\Phi \in C_{0}\left(R^{m m}\right)$ as the family $\left(\nu_{x}\right)_{x \in \Omega}$, that means

$$
<A v\left(\nu_{x}\right)_{x \in \Omega} ; \Phi>=\frac{1}{\text { meas } \Omega} \int_{\Omega} \int_{R^{n+m}} \Phi(v) d \nu_{x} d x .
$$

THEOREM 2.10 (The averaging principle). - Let $\Omega$ be a nonempty, bounded and open subset of $R^{n}$ with meas $(\partial \Omega)=0$. Let $\left(\nu_{x}\right)_{x \in \Omega}$ be a gradient p-Young measure and assume that its underlving deformation $u_{0} \in \xi_{0}+W_{0}^{1, p^{\prime}}\left(\Omega ; R^{m}\right)\left(\xi_{0} \in M^{m \times n}\right)$. Then the average $A v\left(\nu_{x}\right)_{x \in \Omega}$ is a homogeneous gradient p-Young measure with the centre of mass at $\xi_{0}$.

Further we will need two more auxiliary results based on general results from Radon measures theory.

It is well known that the space $C_{0}\left(R^{l}\right)$ with uniform norm has dual space isomorphic to the space of all Radon measures with total variation $\|\cdot\|_{M}$ as a norm, where duality is given by

$$
<\Phi(v): \nu>=\int_{R^{\prime}} \Phi(v) d \nu .
$$

By Banach-Alaoglu theorem a set of all Radon measures, total variation of which does not exceed a fixed value, is weakly* compact. Since $C_{0}\left(R^{l}\right)$ is separable any such set with the topology induced from weak* one is metrizable (cf. [29], Th. 3.15-3.17). It is clear also that the total variation is lower semicontinuous functional with respect to weak* convergence of sequences of Radon measures.

Proposition 2.11. - Let $p \in] 1 . \infty], \nu_{k}(k \in N)$ be homogeneous gradient p-Young measures for which

$$
\int_{R^{\prime \prime \prime}}|v|^{p} d \nu_{k} \leq C<\infty, k \in N
$$

(supports of $\nu_{k}$ are uniformly bounded in the case $p=\infty$ ).

Then, possibly for a subsequence, $\nu_{k} \rightarrow^{*} \nu$ and $\nu$ is also a homogeneous gradient p-Young measure.

A similar assertion has been proved in $[20 ; \operatorname{Pr} .1 .3, \operatorname{Pr} .2 .4]$ in the case $\nu$ is a probability measure and $\nu_{k}$ converges to $\nu$ over all functionals of the form $<\Phi ; \cdot>$, where $\lim _{v \rightarrow \infty} \Phi(v) /|v|^{p}$ exists.

Proof. - Obviously $\nu_{k}$ contains a weak* convergent subsequence (not relabelled) and its limit $\nu$ is a nonnegative measure. Moreover, $\|\nu\|_{M} \leq 1$. In order to prove that $\nu$ is a probability measure it is enough to obtain that $\|\nu\|_{M}=1$. 
Otherwise for some $\delta>0$ we have that $\|\nu\|_{M} \leq 1-\delta$. From (2.1) we infer that $\nu_{k}\left(R^{n m} \backslash B(0, r)\right) \leq C / r^{p}, k \in N$. Hence $\nu_{k}(B(0, r)) \geq 1-\delta / 2$ for sufficiently large $r$ and all $k$. Consider a nonnegative continuous function $\Phi: R^{n m} \rightarrow R$ with compact support and such that $\Phi \leq 1$ everywhere, $\Phi=1$ on $B(0, r)$. Then

$$
\left.\liminf _{k}\left\langle\Phi ; \nu_{k}\right\rangle \geq 1-\delta / 2\right\rangle\langle\Phi ; \nu\rangle
$$

This contradiction proves that $\nu$ is a probability measure.

I.et $\Omega$ he an open bounded set with meas $(\partial \Omega)=0$. For any $k$ there exists a sequence $u_{j}^{k} \in W^{1, \nu}\left(\Omega ; R^{m}\right)$ (k: is fixed) such that $\nabla u_{j}^{k}$ generates $\nu_{k}$ as gradient $p$-Young measure. Consider a countable set of measurable subsets $X$ of $\Omega$ which is dense in the following sense: for any $\epsilon>0$ and a measurable subset $E$ of $\Omega$ there exists $G \in X$ such that the inequality moas $\{(E \backslash G) \cup(G \backslash E)\} \leq \epsilon$ holds. Consider also a countable dense subset $Y$ of $C_{0}\left(R^{n m}\right)$.

We may isolate a sequence $v^{k}=u_{j(k)}^{k}(j(k) \rightarrow \infty$ as $k \rightarrow \infty)$, which is bounded in $W^{1, \nu}\left(\Omega ; R^{m}\right)$, such that

$$
\int_{G} \Phi\left(\nabla v_{k}(x)\right) d x \rightarrow \int_{G} \int_{R^{n m}} \Phi(v) d \nu d x \text { for any } G \in X . \Phi \in Y
$$

(boundedness of $v_{k}$ in $W^{1, p}\left(\Omega ; R^{m}\right)$ follows from (2.1) in the case $p \in] 1, \infty[$, and from Theorem 1.5 of [18] if $p=\infty)$ ).

Hence

$$
\Phi\left(\nabla v_{k}\right) \rightarrow^{*} \int_{R^{n \cdots}} \Phi(v) d \nu \quad \text { in } L^{\infty}(\Omega), \Phi(v) \in C_{0}\left(R^{n m}\right) .
$$

Therefore $\nu$ is generated by gradients of a sequence equi-bounded in $W^{1, p}\left(\Omega ; R^{m}\right)$. By Remark $2.7^{\prime} \nu$ is homogeneous gradient $p$-Young measure.

Proof is completed.

PROPOSITION 2.12. - Let $\Omega$ be a compact set in $R^{n}, V: x \rightarrow V(x)$ be a multivalued mapping, where $V(x)(x \in \Omega)$ are subsets of a set of Radon measures over $R^{l}$ with equi-bounded total variations. Suppose also that the mapping $V$ satisfies the following requirements:

1. For each $x \in \Omega V(x)$ is nonempty set, which is closed with respect to weak* convergence,

2. $V$ is upper semicontinuous with respect to weak* convergence: for any $x_{k} \rightarrow x_{0}$ and $\nu_{k} \in V\left(x_{k}\right)$ such that $\nu_{k} \rightarrow^{*} \nu_{0}$ we have that $\mu_{0} \in V\left(x_{0}\right)$.

Then $V$ admits a selection $\left(\nu_{x}\right)_{x \in \Omega} \in C_{0}\left(R^{l}\right)^{\prime}$. 
Proof. - Consider a set $\tilde{V}$ of all Radon measures, total variation of which does not exceed a fixed value, such that $V(x) \subset \tilde{V}$ for all $x \in \Omega$. As it was mentioned above $\hat{V}$ is a complete metric space in the topology induced from weak* one.

Therefore $V$ is a closed, nonempty and upper semicontinuous multivalued mapping with values in a complete metric space. By Theorem on measurable selection [23] there exists a measurable selection of $V$, where measurability means that the preimage of any closed set is measurable. It is clear that such a family of measures has measurable action on elements of $C_{0}\left(R^{l}\right)$.

It completes the proof.

\section{NEW PROOFS OF THEOREMS 1.5, 1.6. PROOF OF 'THEOREM 1.8.}

We start this section with the proof of Theorem 1.5 which may be obtained through results from nongradient Young measure theory mentioned in $\$ 2$ (Propositions 2.2-2.5) and Lemma 3.1 stated below. The proof of the theorems 1.6, 1.8 is more complicated and will involve some additional auxiliary results and the results on gradient Young measures from $\xi 2$.

LEMma 3.1. - Let $L(v): R^{l} \rightarrow R$ be a continuous function, 1 be a probability measure with the centre of mass at $v_{0} \in R^{l}$.

1. If $L(v)$ is convex at $v_{0}$ then $\int_{R^{i}} L(v) d v \geq L\left(v_{0}\right)$.

2. If $L(v)$ is strictly convex at $v_{0}$ and $\nu \neq \delta_{r_{0}}$ then $\int_{R^{\prime}} L(v) d \nu>L\left(v_{0}\right)$.

Proof. - In view of convexity of $L$ at $v_{0}$ there exists $g \in \partial L\left(v_{0}\right)$ (cf. Lemma 2.1 from [30]). Therefore

$$
\int_{R^{\prime}}\left\{L(v)-L\left(v_{0}\right)\right\} d v \geq \int_{R^{\prime}}\left\langle g, v-v_{0}\right\rangle d \nu=0 .
$$

Let $L(v)$ be strictly convex at $v_{0}$ and $\nu \neq \delta_{v_{0}}$. Denote by $M(\nu)$ the affine hull of supp $\nu$ and suppose that $\operatorname{dim} M(\nu) \geq 1$. The restriction of $L(v)$ to $M(\nu)$ is strictly convex at $v_{0}$ on $M(\nu)$. By Lemma 2.2 from [30] there exists $e \in\left\{v-v_{0}: v \in M(\nu)\right\}(|e|=1)$ such that

$$
L(v)-L\left(v_{0}\right)-<g \cdot v-v_{0}>>0
$$

if $v \in M(\nu)$ and $<v-v_{0}, e><0$. 
Hence the equality $\int_{R^{l}} L(v) d \nu=L\left(v_{0}\right)$ implies that

$$
\operatorname{supp} \nu \bigcap\left\{v \in M(\nu):<v-v_{0}, e><0\right\}=\emptyset .
$$

Because $v_{0}$ is the centre of mass for $\nu$ we get also that

$$
\operatorname{supp} \nu \subset\left\{v \in M(\nu):<v-v_{0}, e>=0\right\} .
$$

It contradicts the definition of $M(\nu)$.

Hence $\operatorname{dim} M(\nu)=0$, that is $\nu=\delta_{v_{0}}$.

It completes the proof.

Proof of Theorem 1.5. - Without loss of generality we may suppose that $\liminf _{k \rightarrow \infty} I\left(u_{k}, \xi_{k}\right)<\infty$. In view of Theorem 2.2 we obtain that any Young measure generated by a subsequence of $\left(u_{k}, \xi_{k}\right)$ is of the form $\left(\delta_{u_{0}(x)} \times \nu_{x}\right)_{x \in \Omega}$. By Proposition 2.5 the inequality

$$
\liminf _{k \rightarrow \infty} I\left(u_{k}, \xi_{k}\right) \geq \int_{\Omega} \int_{R^{\prime}} L\left(x, u_{0}(x), v\right) d \nu_{x} d x
$$

holds.

If for a.e. $x \in \Omega L\left(x, u_{0}(x), v\right)$ is convex at $v=\xi_{0}(x)$ then by Lemma 3.1 we have that

$$
L\left(x, u_{0}(x), \xi_{0}(x)\right) \leq \int_{R^{i}} L\left(x, u_{0}(x), v\right) d \nu_{x} \text { for a.e. } x \in \Omega .
$$

Hence

$$
\liminf _{k \rightarrow \infty} I\left(u_{k}, \xi_{k}\right) \geq I\left(u_{0}, \xi_{0}\right),
$$

that proves the first statement of the theorem. Moreover, the convergence $I\left(u_{k}, \xi_{k}\right) \rightarrow I\left(u_{0}, \xi_{0}\right)<\infty$ implies equality

$$
L\left(x, u_{0}(x), \xi_{0}(x)\right)=\int_{R^{t}} L\left(x, u_{0}(x), v\right) d \nu_{x} \text { for a.e. } x \in \Omega .
$$

An additional condition of strict convexity of $L\left(x, u_{0}(x), v\right)$ at $v=\xi_{0}(x)$ for a.a. $x \in \Omega$ gives that in this case $\nu_{x}=\delta_{\xi_{0}(x)}$ for a.e. $x \in \Omega$ (see Lemma 3.1). Hence $\xi_{k} \rightarrow \xi_{0}$ in $L^{1}$ by Proposition 2.3. The rest is a consequence of this fact and Proposition 2.5. The proof of the theorem is completed. 
COROLlary 3.2. - Let $L: R^{n} \rightarrow R$ be a continuous function and $v_{0} \in R^{n}$. The integral functional

$$
J(u)=\int_{\Omega} L(\nabla u(x)) d x
$$

is weak slsc in $W^{1,1}(\Omega)$ and weak* slsc in $W^{1, \infty}$ at the function $\left\langle v_{0}, \cdot\right\rangle$ simultaneously. Moreover any of these properties holds if and only if $L(v)$ is convex at $v_{0}$ in the sense of Definition 1.4.

Let $L(v)$ be convex at $v_{0}$. The weak-strong convergence property at the function $\left\langle v_{0} \cdot \cdot>\right.$ over $W^{1,1}(\Omega)$ and $W^{1, \infty}(\Omega)$ holds simultaneously and is equivalent to strict convexity of $L(v)$ at $v_{0}$ given by Definition 1.4.

The second part of the assertion is a result of the paper [10].

$$
\text { Proof. - If } L(v) \text { is convex at } v_{0} \text { then for a }\left.g \in \partial L(v)\right|_{v=v_{0}}
$$

$$
L(v)-L\left(v_{0}\right)-<g, v-v_{0}>\geq 0, v \in R^{n} .
$$

The functional $\int_{\Omega}<g, \nabla u(x)>d x$ is continuous with respect to weak convergence of sequences in $W^{1,1}(\Omega)$. These observations together give the lower semicontinuity result.

Let us prove now that weak ${ }^{*}$ sequential lower semicontinuity in $W^{1, \infty}(\Omega)$ of the functional at the function $\left\langle v_{0}, \cdot\right\rangle$ implies convexity of $L(v)$ at $v_{0}$. Otherwise we may introduce $c_{i} \geq 0, v_{i} \in R^{n}$ for which $\sum_{i} c_{i}=1$, $\sum_{i} c_{i} L\left(v_{i}\right)<L\left(v_{0}\right)$. Consider the probability measure $\nu=\sum_{i} c_{i} \delta_{v_{i}}$. This measure is a homogeneous gradient Young measure with the underlying deformation $\left\langle v_{0}, \cdot\right\rangle$. Actually, the requirements (i), (iii) from Theorem 2.7 are satisfied automatically (case $p=\infty$ ), (ii) holds because in the scalar case quasiconvexity is just convexity implying, as a consequence, validity of Jensen inequality for any probability measure [13, Ch. 10]. Hence, the functional is not lower semicontinuous on a sequence associated with $\nu$.

We proved that convexity at $v_{0}$ is necessary condition for an integral functional to be sequential weak* in $W^{1, \infty}(\Omega)$ lower semicontinuous at the function $\left\langle v_{0}, \cdot\right\rangle$.

The proof is similar for the weak-strong convergence property.

Strict convexity of $L(v)$ at $v_{0}$ provides the weak-strong convergence property at $\left\langle v_{0} \cdot \cdot\right\rangle$ by Theorem 1.5. The lack of strict convexity of $L(v)$ at $v_{0}$ leads to the lack of the weak-strong convergence property at $\left.<v_{0}, \cdot\right\rangle$ by arguments analogous to ones introduced above. Actually, in this case $\sum_{i} c_{i} L\left(v_{i}\right)=L\left(v_{0}\right)$ for some $c_{i} \geq 0, v_{i} \neq v_{0}$ such that $\sum_{i} c_{i}=1, \sum_{i} c_{i} v_{i}=v_{0}$. Hence $\sum_{i} c_{i} \delta_{v_{i}}$ is a gradient Young measure with the center of mass at $v_{0}$, and for a sequence associated with this measure the weak-strong convergence property does not hold. 
The proof of the corollary 3.2 is completed.

The arguments of the proof to Theorem 1.8 will involve some intermediate constructions which have been introduced as objects of independent interest in $[20, \S 6.3],[28],[21]$.

Definition 3.3. - Let $L(v): R^{n m} \rightarrow R$ be a continuous function. $L(v)$ is closed p-quasiconvex at a point $v_{0} \in R^{n m}$ if

$$
\int_{R^{\prime \prime \prime \prime}} L(v) d \nu \geq L\left(v_{0}\right)
$$

for any homogeneous gradient p-Young measure $\nu$ with underlying deformation $v_{0}$.

$L(v)$ is strictly closed p-quasiconvex at $v_{0}$ if additionally the strict inequality always holds for $\nu \neq \delta_{v_{0}}$.

There exist direct connections between quasiconvexity (strict $p$-quasiconvexity) and closed $p$-quasiconvexity (strict closed $p$-quasiconvexity).

Proposition 3.4. - If $L: R^{n m} \rightarrow R$ is continuous, $|L(v)| \leq A|v|^{p}+B$, $A, B>0, p \in[1, \infty[(L$, is only continuous in the case $p=\infty)$ then $L$ is quasiconvex at $v_{0}$ if and only if it is closed p-quasiconvex at this point. Moreover, strict p-quasiconvexity at a point implies strict closed pquasiconvexity at the same point, and the converse is true at least under additional requirements of boundedness below of integrands and $p \in] 1, \infty]$.

Proof. - If $\nu$ is a homogeneous gradient $p$-Young measure with the underlying deformation $v_{0}$. then by Proposition 2.8 it is generated by gradients of a sequence $v_{0} \cdot+\phi_{k}$ where $\phi_{k} \in C_{0}^{\infty}\left(\Omega ; R^{m}\right)$ are such that $\left|\nabla \phi_{k}\right|^{p}(k \in N)$ are equi-integrable. Having the inequality

$$
\int_{\Omega} L\left(v_{0}+\nabla \phi_{k}\right) d x \geq L\left(v_{0}\right) \text { meas } \Omega
$$

for any $k$ we come to the inequality $\int_{R^{n m}} L(v) d \nu \geq L\left(v_{0}\right)$ meas $\Omega$ through Proposition 2.5 in view of the growth conditions on $L$ and equi-integrability of $\left|\nabla \phi_{k}\right|^{p}$. Hence quasiconvexity implies its closed analogy.

If $L$ is strictly $p$-quasiconvex at $v_{0}$ then the equality

$$
\lim _{k \rightarrow \infty} \int_{\Omega} L\left(v_{0}+\nabla \phi_{k}\right) d x=L\left(v_{0}\right) \text { meas } \Omega
$$

holds for a sequence $\phi_{k}$ bounded in $W_{0}^{1, p}\left(\Omega ; R^{m}\right)$ only if $\nabla \phi_{k} \rightarrow 0$ in measure. It proves that strict $p$-quasiconvexity implies its closed analogy. 
Conversely, if $L$ is closed $p$-quasiconvex at $v_{0}$ and $\phi \in W_{0}^{1, \infty}\left(\Omega ; R^{m}\right)$ then

$$
\int_{\Omega} L\left(v_{0}+\nabla \phi\right) d x=\int_{R^{\prime \prime \prime n}} L(v) d A v\left(\delta_{v_{0}+\nabla_{\phi}}\right) \text { meas } \Omega \geq L\left(v_{0}\right) \text { meas } \Omega,
$$

that means quasiconvexity at $v_{0}$.

Let $L(v)$ be strictly closed $p$-quasiconvex at $v_{0}(p>1)$ and bounded below. Assume that $L(v)$ is not strictly $p$-quasiconvex at $v_{0}$. Then for a sequence $\phi_{k} \in C_{0}^{\infty}\left(\Omega ; R^{m}\right)$ bounded in $W^{1, p}\left(\Omega ; R^{m}\right)$ and some $\varepsilon>0$ we have that

$$
\int_{\Omega} L\left(v_{0}+\nabla \phi_{k}\right) d x \rightarrow L\left(v_{0}\right) \operatorname{meas} \Omega, k \rightarrow \infty .
$$

where $\operatorname{meas}\left\{x \in \Omega:\left|\nabla \phi_{k}(x)\right|>\varepsilon\right\}>\varepsilon \operatorname{meas} \Omega$.

The averages $\nu_{k}$ of $\delta_{v_{0}}+\nabla \phi_{k}$, have the properties

$$
\begin{gathered}
\int_{R^{\prime m m}}|v|^{p} d \nu_{k} \leq C<\infty, k \in N, \\
\lim _{k \rightarrow \infty} \int_{R^{n m}} L(v) d \nu_{k}=L\left(v_{0}\right), \\
\nu_{k}\left(R^{n m} \backslash B\left(v_{0}, \varepsilon\right)\right) d x \geq \varepsilon, k \in N .
\end{gathered}
$$

A weak ${ }^{*}$ limit $\nu$ of a subsequence of $\nu_{k}$ is a gradient $p$-Young measure with the centre of mass at $v_{0}$ due to Proposition 2.11. Hence the inequality

$$
\int_{R^{n, n}} L(v) d \nu \geq L\left(v_{0}\right)
$$

is valid. The converse inequality together with the inequality

$$
\nu\left(R^{n m} \backslash B\left(v_{0}, \varepsilon / 2\right)\right) d x \geq \varepsilon
$$

are direct consequences of weak* convergence and boundedness below of $L$.

We come to a contradiction, which completes the proof.

Remark. - It is clear from arguments of the proof that strict closed $p$-quasiconvexity still implies strict $p$-quasiconvexity if

$$
-A|v|^{r}-B \leq L(v) \leq A|v|^{p}+B, A, B>0, r>p .
$$

The same is not clear if $r=p$ (probably here exist counterexamples; see also Remark 3.5). 
Proof of Theorem 1.8. - 1. We will reduce proof of "sufficient" part of the theorem through Proposition 3.4 to arguments introduced in [20, \$6.3], [28], [21]. Let $L(x, u(x), v)$ be quasiconvex at $v=\nabla u_{0}(x)$ correspondingly for a.e. $x \in \Omega$, and $u_{k}$ be an arbitrary sequence weakly convergent to $u_{0}$ in $W^{1, p}\left(\Omega ; R^{m}\right)$, for which negative parts of $L\left(x, u_{k}, \nabla u_{k}\right)$ are equiintegrable. Without loss of generality we may suppose that the limit of $I\left(u_{k}\right)$ exists, and that the sequence $\left(u_{k}(x), \nabla u_{k}(x)\right)$ generates a family of measures $\left(\delta_{u_{0}(x)} \times \nu_{x}\right)_{x \in \Omega}$, where $\left(\nu_{x x}\right)_{x \in \Omega}$ satisfies all requirements (i)-(iii) of Theorem 2.7.

By Proposition 2.5

$$
\lim _{k \rightarrow \infty} I\left(u_{k}\right) \geq \int_{\Omega}\left(\int_{R^{\prime \prime m}} L\left(x, u_{0}(x), v\right) d \nu_{x}\right) d x .
$$

In view of the localization principle 2.9 and Proposition 3.4 the term in the brackets in the right-hand side is minorized by $L\left(x, u_{0}(x), \nabla u_{0}(x)\right)$ for a.e. $x \in \Omega$. It gives the lower semicontinuity result.

Moreover, if $u_{k}$ does not converge to $u_{0}$ in $W^{1,1}\left(\Omega ; R^{m}\right)$ and for a.e. $x \in \Omega L\left(x, u_{0}(x), v\right)$ is strictly $p$-quasiconvex at $v=\nabla u_{0}(x)$ then for every $x$ from a set of positive measure $\nu_{r}$ is not Dirac mass (cf. Proposition 2.3) and, as a consequence, the strict inequality

$$
\int_{R^{n ! m}} L\left(x, u_{0}(x), v\right) d \nu_{x}>L\left(x, u_{0}(x), \nabla u_{0}(x)\right)
$$

holds for these $x$. Therefore the convergence $I\left(u_{k}\right) \rightarrow I\left(u_{0}\right)$ implies strong convergence of $u_{k}$ to $u_{0}$ in $W^{1,1}\left(\Omega ; R^{m}\right)$. By Proposition 2.5 we obtain also that in this case $L\left(x, u_{k}(x), \nabla u_{k}(x)\right) \rightarrow L\left(x, u_{0}(x), \nabla u_{0}(x)\right)$ in $L^{1}$.

The "sufficient" statement of the theorem 1.8 is proved. Let us prove the "necessary" one.

2. Suppose that $I(u)$ is swlsc in $W^{1, p}\left(\Omega ; R^{\prime \prime}\right)$ at $u_{0}$ for all $u_{k} \in$ $u_{0}+C_{0}^{\infty}\left(\Omega ; R^{m}\right)$ with equi-integrable $\left|\nabla u_{k}\right|^{p}$.

By Lusin theorem there exists a sequence of compact sets $\Omega_{k}$ such that meas $\left(\Omega \backslash \Omega_{k}\right) \rightarrow 0$, restrictions of $u_{0}$ and $\nabla u_{0}$ to $\Omega_{k}$ are continuous. We may assume also that the restriction of $L(x, u, v)$ to $\Omega_{k} \times R^{m} \times R^{n m}$ is continuous. Let $k$ be fixed. If for a Lebesgue point $x_{0} \in \Omega_{k} L\left(x_{0}, u_{0}\left(x_{0}\right), v\right)$ is not quasiconvex at $v=\nabla u_{0}\left(x_{0}\right)$ then by Proposition 3.4 for some homogeneous gradient $p$-Young measure with the centre of mass at $\nabla u_{0}\left(x_{0}\right)$ and $\varepsilon>0$ we obtain that

$$
\int_{R^{n} m} L\left(x_{0}, u_{0}\left(x_{0}\right), v\right) d \nu<L\left(x_{0}, u_{0}\left(x_{0}\right), \nabla u_{0}\left(x_{0}\right)\right)-\varepsilon .
$$


The same inequality holds for all $x \in \Omega_{k}$ sufficiently close to $x_{0}$ with $\nu(x)$ obtained from $\nu$ only by exchanging the center of mass from $\nabla u\left(x_{0}\right)$ to $\nabla u(x)$ (if $\nabla u_{j}$ generates $\nu$ as a gradient $p$-Young measure then the measure $\nu(x)$ is generated by $\left.\nabla u_{j}+\nabla u_{0}(x)-\nabla u_{0}\left(x_{0}\right)\right)$. Denote this set as $\Omega_{x_{0}}$. Define $\nu_{x}$ as $\delta_{\nabla u_{0}(x)}$ for all $x \in \Omega \backslash \Omega_{\left.x_{0}\right)}$, and as $\nu(x)$ otherwise. It is easy to verify that all conditions (i)-(iii) of Theorem 2.7 hold, that means $\left(\nu_{n}\right)_{x \in \Omega}$ is a gradient $p$-Young measure. In view of Proposition 2.8 there exists a sequence $\phi_{k} \in C_{0}^{\infty}\left(\Omega ; R^{\prime \prime \prime}\right)$ weakly convergent in $W^{1 . p}\left(\Omega ; R^{\prime \prime \prime}\right)$ to zero and such that $\nabla\left(u_{0}+\phi_{k}\right)$ generates $\left(u_{x}\right)_{x \in \Omega}$ and the functions $\left|\nabla \phi_{k}\right|^{p}$ are equi-integrable. Hence $\lim _{k \rightarrow x} I\left(u_{0}+\phi_{k}\right)<I\left(u_{0}\right)$. A contradiction by which we prove that for a.e. $x \in \Omega L\left(x, u_{0}(x), v\right)$ is quasiconvex at $t=\nabla u_{0}(x)$ correspondingly.

3. We will prove now the last claim of the theorem. Consider the set

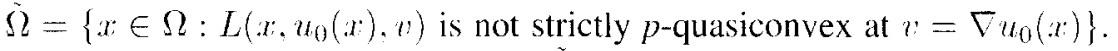
Our purpose is to prove that meas $\check{\Omega}=0$.

Let $\Omega_{k} \subset$ int $\Omega(k \in N)$ be an increasing sequence of compact sets for cvery of which the restrictions of $u_{0}, \nabla u_{0}$ to $\Omega_{k}$ are continuous, the restriction of $L(x, u, v)$ to $\Omega_{k} \times R^{m} \times R^{m m}$ is also continuous and $\operatorname{meas}\left(\Omega \backslash \Omega_{k}\right) \rightarrow 0$ as $h \rightarrow \infty$.

For given $k \in N, \epsilon>0$ consider a set $\Omega_{k, \epsilon} \subset \Omega_{k}$ of $x$, for every of which there exists a gradient homogeneous $p$-Young measure 1 with the centre of mass at $\nabla u_{0}(x)$ and such that

$$
\begin{gathered}
\int_{R^{n \prime \prime}} L\left(x, u_{0}(x), v\right) d \nu=L\left(x, u_{0}(x), \nabla u_{0}(x)\right), \\
\int_{R^{n+m}}|v|^{p} d \nu \leq 1 / \epsilon \\
\nu\left(B\left(\nabla u_{0}(x), \epsilon\right)\right) \leq 1-\epsilon .
\end{gathered}
$$

We will prove that $\Omega_{k, f}$ is a closed set. Moreover the multivalued mapping $V: x \rightarrow V(x)$, where $V(x)$ is the set of all probability measures satisfying the above requirements, admits a selection $(\nu(x))_{x \in \Omega_{k,},} \in C_{0}\left(R^{m m}\right)^{\prime}$. These results are enough to complete the proof. Actually, if meas $\Omega_{k, t}>0$ then the parametrized measure $\left(\nu_{x}\right)_{x \in \Omega}$, which is equal to $\nu(x)$ for $x \in \Omega_{k, \epsilon}$, and to $\delta_{\nabla u_{0}(x)}$ for other $x \in \Omega$, satisfies all requirements of Theorem 2.7 . Therefore $\left(\nu_{x}\right)_{x \in \Omega}$ is a gradient $p$-Young measure. In view of (3.3) this measure is not a Dirac mass for a set of positive measure, and by (3.1)

$$
\int_{\Omega} \int_{R^{n m}} L\left(x, u_{0}(x), v\right) d \nu_{x} d x=\int_{\Omega} L\left(x, u_{0}(x), \nabla u_{0}(x)\right) d x
$$


In view of Proposition 2.8 the weak-strong convergence property fails on a sequence $u_{k}$ corresponding to $\left(\nu_{x}\right)_{x \in \Omega}$. It gives a contradiction. Therefore meas $\Omega_{k, \epsilon}=0$ for any $k, \epsilon$. Hence meas $\tilde{\Omega}=0$ too.

Start now the proof of the desired properties of $V$ with closedness for $V(x)$, where $x \in \Omega_{k,+}$ is fixed. Consider a sequence $\nu_{k} \in V(x)$ weakly* convergent to $\nu$. By Proposition $2.11 \nu$ is a homogeneous gradient

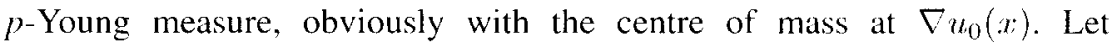
$\Phi_{j}(v) \in C_{0}^{\infty}\left(R^{m m}\right)(j \in N)$ be nonnegative functions such that for any $v \in R^{n m} \Phi_{j}(v) \neq 0$ only for a finite set of $j \in N$ and $\sum_{i=1}^{\infty} \Phi_{j}(v)=1$.

Then

$$
\begin{gathered}
\lim _{k \rightarrow \infty} \int_{R^{\prime \prime \prime m}}|v|^{\prime \prime} d \nu_{k}=\lim _{k \rightarrow \infty} \int_{R^{\prime \prime m}} \sum_{j} \Phi_{j}(v)|v|^{\prime \prime} d \nu_{k} \geq \\
\sum_{j} \lim _{k \rightarrow \infty} \int_{R^{n+m}} \Phi_{j}(v)|v|^{p} d \nu_{k}=\int_{R^{\prime \prime m}} \sum_{j} \Phi_{j}(v)|v|^{\prime} d \nu=\int|v|^{p} d \nu .
\end{gathered}
$$

Therefore $\nu$ satisfies (3.2). Morcover, $\nu$ satisfics also (3.3). Otherwise for some $\tilde{\epsilon}<\epsilon$ we obtain $\nu\left(B\left(\nabla u_{0}(x), \tilde{\epsilon}\right)\right)>1-\epsilon$. For a nonnegative function $\Phi(v): R^{m m} \rightarrow R$ with the support in $B\left(\nabla u_{0}(x) . \epsilon\right)$ and equal to 1 on $B\left(\nabla u_{0}(x), \tilde{\epsilon}\right)$ we obtain that

$$
1-\epsilon \geq \limsup _{k \rightarrow \infty} \int_{R^{n+m}} \Phi(v) d \nu_{k}=\int_{R^{n+1}} \Phi(v) d \nu>1-\epsilon .
$$

A contradiction.

The last step is to prove validity of (3.1) for $\%$. The right hand side does not exceed the left one because $\nu$ is gradient $p$-Young measure and $L\left(x, u_{0}(x), v\right)$ is quasiconvex at $v=\nabla u_{0}(x)$. Converse inequality may be proved by the same arguments as (3.2) has been proved in view of boundedness below of $L$.

We have proved that $V: \Omega_{k, \epsilon} \rightarrow 2^{M_{1}^{+}}$is closed multivalued mapping. In order to obtain upper semicontinuity for $V$ and closedness for $\Omega_{k_{i},}$ we need to prove that for $x_{k} \rightarrow x_{0}, \nu_{k} \rightarrow^{*} \nu$ such that $\nu_{k}$ satisfy (3.1)-(3.3) (with $x_{k}$. instead $x$ ) the same is true for $\nu$ (with $x_{0}$ instead $x$ ). But the proof is nothing more than repetition of the arguments utilized in the proof for closedness of $V(x)$ in view of continuity of the restriction of $L$ to $\Omega_{k, \epsilon} \times R^{m} \times R^{n m}$.

It completes the proof.

Remark 3.5. - One should mention that the last assertion of the theorem 1.8 is valid under a less restrictive assumption on $L(x, u, v)$ :

$$
-A|v|^{r}-B \leq L \leq A|v|^{p}+B . A, B>0, r>p .
$$

Vol. 15, n $6-1998$. 
Actually, under this requirement the right hand side inequality in (3.1) still holds for the limit measure 1 . The same is not clear for $r=p$ (see also Remark to the proof of Proposition 3.4).

In the general case $\left(|L| \leq A|v|^{p}+B\right)$ it is possible to prove that the weak-strong convergence property at $u_{0} \in W^{1 . p}\left(\Omega: R^{m}\right)$ implies that either for a.e. $x \in \Omega L\left(x, u_{0}(x), v\right)$ is strictly $r$-quasiconvex at $v=\nabla u_{0}(x)$ for any $r>p$ or the same property holds for the family $L\left(x \cdot u_{0}(x), v\right)$.

Actually, in this case the weak-strong convergence property implies that either for a.e. $x \in \Omega$ the function $L\left(x, u_{0}(x), v\right)$ is quasiconvex at $v=\nabla u_{0}(x)$ or this property holds for the family $-L\left(x, u_{0}(x), v\right)$. Otherwise, following arguments from Part 2 of the proof, we may introduce $x_{1} \neq x_{2}$, which are Lebesgue points of $\Omega_{k}$ for some $k$, such that for their neighbourhoods $B_{x_{1}}, B_{x_{2}}$ in $\Omega_{k_{1}}\left(B_{x_{1}} \cap B_{x_{2}}=()\right)$ there exist nontrivial $p$-Young measures $\nu_{x}^{1} \cdot \nu_{x}^{2}$, with the centers of mass at $\nabla u_{0}(x)$, satisfying conditions (ii), (iii) of Theorem 2.7 and the inequalities

$$
\begin{aligned}
& \int_{R^{m}} L\left(x, u_{0}(x), v\right) d \nu_{x:}^{1}<L\left(x, u_{0}(x) . \nabla u_{0}(x)\right)-\varepsilon \text {. if } x \in B_{x 1} \text {, } \\
& \int_{R^{\prime \prime m}} L\left(x, u_{0}(x), v\right) d \nu_{, r}^{2}>L\left(x, u_{0}(x) . \nabla u_{0}(x)\right)+\varepsilon, \text { if } x \in B_{r_{2}} .
\end{aligned}
$$

Exchanging, if nccessary, ncighbourhoods $B_{x_{1}}, B_{x_{2}}$ we may achicve also the equality

$$
\begin{gathered}
\int_{B_{x,},} \int_{R^{n+1,}} L\left(x, u_{0}(x), v\right) d \nu_{x}^{1} d x+\int_{B_{x_{2}}} \int_{R^{\prime \prime \prime}} L\left(x, u_{0}(x), v\right) d \nu_{x}^{2} d x= \\
\int_{B x_{1} \cup B x_{2}} L\left(x, u_{0}(x), \nabla u_{0}(x)\right) d x .
\end{gathered}
$$

Consider a $p$-Young measure $\left(\nu_{x}\right)_{x \in \Omega}$ which is equal to $\nu_{x}^{1}$ on $B_{x_{1}}, \nu_{x}^{2}$ on $B_{x_{2}}$, and $\delta_{\nabla u_{0}(x)}$ for other points of $\Omega$. It is easy to verify validity of conditions (i)-(iii) from Theorem 2.7 that means $\left(\nu_{x}\right)_{x \in \Omega}$ is a gradient p-Young measure.

By Proposition 2.8 there exists a sequence $u_{k} \in u_{0}+C_{0}^{\infty}\left(\Omega ; R^{m}\right)$ such that $\left|\nabla u_{k}\right|^{p}$ are equi-integrable and $\nabla u_{k}$ generates $\left(\nu_{x}\right)_{x \in \Omega}$. Then $I\left(u_{k}\right) \rightarrow I\left(u_{0}\right), u_{k}$ converges to $u_{0}$ in $W^{1, p}\left(\Omega ; R^{m}\right)$ only weakly. The weak-strong convergence property fails at this sequence. A contradiction.

Therefore either $L\left(x, u_{0}(x), v\right)$ is quasiconvex at $v=\nabla u_{0}(x)$ for a.a. $x \subset \Omega$ or the same holds for $\cdots L\left(x, u_{0}(x), v\right)$. Without loss of generality we may suppose that this property is valid for $L\left(x, u_{0}(x), v\right)$. Then strict 
$r$-quasiconvexity for $r>p$ may be proved by the arguments from Part 3 of the proof because in this case the equality (3.1) holds automatically for any measure, which is weak* limit of measures $\nu_{k}$ satisfying conditions (3.1)-(3.3) and the inequality $\int|v|^{r} d \nu_{k} \leq C<\infty$.

Proof of Theorem 1.6 may be obtained as a consequence of the results of the above remark. By this remark either for a.e. $x \in \Omega L\left(x, u_{0}(x), v\right)$ is strictly $\infty$-quasiconvex at $v=\nabla u_{0}(x)$ or the same property holds for the family $-L\left(x, u_{0}(x), v\right)$. But in the scalar case quasiconvexity and strict $x$-quasiconvexity are correspondingly convexity and strict convexity given by Definition 1.4 (cf. Propositions 1.2, 3.2). It gives the required result.

Remark 3.6. - Propositions 1.2, 3.2 let us assert that in the scalar case quasiconvexity at a point is equivalent to convexity given by Definition 1.4 and, as a consequence, implies swlsc in any space $W^{1, p}(\Omega)$ without any conditions on growth of integrands $L(\nabla u)$.

The situation does not have analogous character in the vector-valued case. Actually, by results from [6] the functional with the quasiconvex integrand $|\operatorname{det} \nabla u|$ (here $m=n$ ) is not sequential weak lower semicontinuous over $W^{1, p}\left(\Omega: R^{n}\right)$ if $p<n$ (see also [26] for additional information).

\section{REMARKS ON THE DEFINITION OF STRICT QUASICONVEXITY. SOME RESULTS ON CHARACTERIZATION OF CLASSES OF FUNCTIONALS HAVING THE WEAK-STRONG CONVERGENCE PROPERTY EVERYWHERE}

As it was mentioned in Introduction the definition of strict $p$ quasiconvexity may seem quite odd. One may suppose that it is more natural to demand only strict inequality

$$
\int_{\Omega} L\left(v_{0}+\nabla \phi(x)\right) d x>L\left(v_{0}\right) \text { meas } \Omega
$$

for all $\phi(x) \in C_{0}^{\infty}\left(\Omega ; R^{m}\right)$ not equal identically to zero.

We have explained some reasons for our choice in Introduction. Moreover, our definition coincides with strict convexity in the scalar case. Actually, due to Corollary 3.2 , in the scalar homogeneous case $\left(L(v): R^{n} \rightarrow R\right.$ ) the weak-strong convergence property holds at the function $\left\langle v_{0}, \cdot>\right.$ (this is equivalent to strict $p$-quasiconvexity of $L(v)$ at $v_{0}$ by Proposition 3.4) if and only if $L(v)$ is strictly convex at $v_{0}$. 
Simultaneously strict inequality in the defining inequality for quasiconvexity does not imply the weak-strong convergence property at $\left\langle v_{0}, \cdot\right\rangle$, and, as a consequence, strict convexity for $L(v)$ at $v_{0}$. To demonstrate it we will utilize recent results of Cellina-Friesecke [8], [9], [15] which give precise conditions on an integrand $L(v)$ for a minimization problem

$$
\int_{\Omega} L(\nabla u(x)) d x \rightarrow \min ,\left.u\right|_{\partial \Omega}=<v_{0}, \cdot>, u \in W^{1,1}(\Omega)
$$

to have a solution. Here $L(v): R^{n} \rightarrow R$ is a nonnegative continuous function. The matter is that for an integrand $L(v)$, which is convex at $v_{0}$, the problem has more than one (which is obviously $\left\langle v_{0}, \cdot\right\rangle$ ) solution if and only if for $\left.l \in \partial L(v)\right|_{v=v_{1}}$ the convex hull of the set

$$
U_{v_{0}}=\left\{v \in R^{n}: L(v)-L\left(v_{0}\right)-<l, v-v_{0}>=0\right\}
$$

contains $v_{0}$ as an interior point. It is clear that in this case $\partial L\left(v_{0}\right)$ contains only one element. Hence, there are no solutions of the problem different from the linear one if $\operatorname{dim}\left\{U_{v_{0}}\right\} \in\{1, \ldots, n-1\}$ (as a consequence the strict inequality in the defining inequality for quasiconvexity holds), but the weak-strong convergence property fails at $\left\langle v_{0}, \cdot\right\rangle$ in view of Corollary 3.2 .

A simple example

$$
L\left(v_{1}, v_{2}\right)=\max \left\{v_{1}^{2}-1 ; 0\right\}+v_{2}^{2}
$$

of the function $L(v): R^{2} \rightarrow R$ just gives a convex integrand of such type. The function $L\left(v_{1}, v_{2}\right)$ is not strictly convex at any point $v$, for which $\left|v_{1}\right|<1$, and is strictly convex at other points. The above mentioned necessary and sufficient condition for existence of more than one solution to the minimization problem is not satisfied for all affine boundary conditions in view of strict convexity of $L(v)$ in $v_{2}$ everywhere. Therefore, in spite of validity of strict inequality in the defining inequality for quasiconvexity for each $v_{0}$ and each nontrivial function $\phi \in W_{0}^{1,1}(\Omega)$, the weak-strong convergence property fails at affine functions $\left\langle v_{0}, \cdot\right\rangle$ such that $\left|\left(v_{0}\right)_{1}\right|<1$.

It is well known that an integral functional $I(u)$ of the general type has sequential weak lower semicontinuity property at each function if and only if $L(x, u, v)$ is quasiconvex in $v$ for a.e. $x$ and each $u$ (cf. Theorem 1.3; see also [25] for a simpler and selfcontained proof in the case $L=L(\nabla u)$ ). 
Moreover,

THEOREM 4.1. - Let $|L(x, u, v)| \leq A|v|^{p}+B(A, B>0 ; p \in[1, \infty[)$ be a Caratheodory integrand such that

$$
\liminf \operatorname{in}_{k \rightarrow \infty} I\left(u_{k}\right) \geq I\left(u_{0}\right)
$$

for every affine function $u_{0}$ and every sequence $u_{k} \in u_{0}+C_{0}^{\infty}\left(\Omega ; R^{m}\right)$ such that $\left|\nabla u_{k}\right|^{p}$ are equi-integrable and $u_{k} \rightarrow u_{0}$ in $W^{1, p}\left(\Omega ; R^{m}\right)$.

Then $L(x, u, v)$ is quasiconvex in $v$ for a.e. $x \in \Omega$ and all $u \in R^{m}$.

Proof. - Let $\Omega_{k}$ be an increasing sequence of compact subsets of int $\Omega$ such that the restrictions of $L(x, u, v)$ to $\Omega_{k} \times R^{m} \times R^{n m}$ are continuous, $\operatorname{meas}\left(\Omega \backslash \Omega_{k}\right) \rightarrow 0$ as $k \rightarrow \infty$.

Let $x_{0}$ be a Lebesgue point of $\Omega_{k}$ ( $k$ is tixed). Consider the affine function $u(x)$ such that $u\left(x_{0}\right)=u_{0}, \nabla u(x) \equiv v_{0}$. By arguments of Part 2 of the proof to Theorem 1.8 we obtain that $I(x, u, \cdot)$ is quasiconvex at $v_{0}$. Therefore $L(x, u, v)$ is quasiconvex in $v$ for any $u \in R^{m}$ and any Lebesgue point $x$ of $\Omega_{k}$.

This proves the theorem.

In spite the situation does not have analogous character for the weak-strong convergence property (cf. Example 4.3 below) similar characterization holds for integrands independent on $u$.

Tilcorcm 4.2. - Let $L(x, v): R^{n} \times R^{n m} \rightarrow R$ be a Caratheodory integrand quasiconvex in $v,-B \leq L(x, v) \leq A|v|^{p}+B$ for some $A, B>0, p \in] 1, \infty[$ (in the case $p=\infty|L(x, v)| \leq g(x,|v|)$ ), where $g$ is nondecreasing in the last argument and integrable in $x$ for a fixed $v)$.

The weak-strong convergence property holds over $W^{1, p}\left(\Omega ; R^{m}\right)$ if and only if $L(x, v)$ is strictly $p$-quasiconvex in $v$ for a.e. $x \in \Omega$.

Proof. - The fact that strict $p$-quasiconvexity implies the weak-strong convergence property was proved in Theorem 1.8. To prove the converse. Consider an increasing sequence of compact sets $\Omega_{k} \subset$ int $\Omega$ such that the restriction of $L(x, v)$ to $\Omega_{k} \times R^{n m}$ is continuous for every $k \in N$ and $\operatorname{meas}\left(\Omega \backslash \cup_{k} \Omega_{k}\right)=0$.

For fixed $k \in N, \varepsilon>0$ consider the set of all $\left(x_{0}, v_{0}, \nu\right)$ satisfying requirements: $x_{0} \in \Omega_{k}, v_{0} \in R^{r m},\left|v_{0}\right| \leq 1 / \varepsilon$, and $\nu$ is a homogeneous gradient $p$-Young measure with the centre of mass at $v_{0}$ such that

$$
\begin{gathered}
\int_{R^{n m}} L\left(x_{0}, v\right) d \nu=L\left(x_{0}, v_{0}\right), \\
\int_{R^{n m}}|v|^{p} d \nu \leq 1 / \varepsilon
\end{gathered}
$$




$$
\nu\left(B\left(v_{0}, \varepsilon\right)\right) \leq 1-\varepsilon
$$

Denote the projection of this set on $\Omega_{k}$ as $\Omega_{k, \epsilon}$. Consider the multivalued mapping $V: x_{0} \in \Omega_{k, \leftarrow} \rightarrow\left(v_{0}, \nu\right)$ defined by (4.1)-(4.3). By arguments analogous to ones introduced in Part 3 of the proof of Theorem 1.8 we establish that $\Omega_{k, z}$ is closed set and the multivalued mapping $V$ is upper semicontinuous: the convergences $x_{k} \rightarrow x_{0}, v_{k} \rightarrow v_{0}$, and $\nu_{k} \rightarrow^{*} \nu_{0}$ of $\left(v_{k}, \nu_{k}\right) \in V\left(x_{k}\right)$ imply that $\left(v_{0}, \nu_{0}\right) \in V\left(x_{0}\right)$.

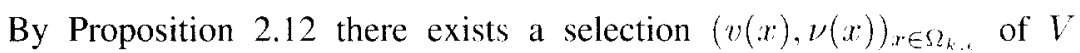
such that $v(x)$ is measurable function, $(\nu(x))_{x \in \Omega_{k .}} \subset C_{0}\left(R^{n m}\right)^{\prime}$. If meas $\Omega_{k, \varepsilon}>0$ then, after restricting (if necessary), we may suppose that $v(x)$ is continuous function defined on a compact subset $K \subset$ int $\Omega$ of positive measure.

By [2] there exists a function $u(x) \in W^{1, p}\left(\Omega ; R^{m}\right)$ such that $\nabla u(x)=$ $v(x)$ on a compacl subset $K_{1} \subset K$ of positive measure. In view of validity of (4.1)-(4.3) for any $x \in K_{1}$ with $v_{0}=v(x), \nu=\nu(x)$ we obtain that for a sequence generating the gradient $p$-Young measure $\left(\nu_{x}\right)_{x \in \Omega}$ (see Proposition 2.8), where $\nu_{x}=\nu(x)$ for $x \in K_{1}$ and $\nu_{x}=\delta_{\nabla u(x)}$ - otherwise, the weak-strong convergence property does not hold.

This contradiction proves that meas $\Omega_{k, \varepsilon}=0$ for any $k, \varepsilon$. It completes the proof by Proposition 3.4 .

Consider the integrand $L(u, v): R \times R^{2} \rightarrow R$ (here $v=\left(v_{1}, v_{2}\right) \in R^{2}$ )

$$
L(u, v)=\max \left\{\left(v_{1}-2\right)^{2}-1: 0\right\}+v_{2}^{2}+u^{2}\left(v_{1}^{2}+v_{2}^{2}\right) .
$$

Proposition 4.3. - The functional $I(u): W^{1,2}(\Omega) \rightarrow R$ with the above introduced integrand admits the weak-strong convergence property everywhere in spite of lack of strict convexity of $L(u, v)$ in v for $u=0$, $\left.v=\left(v_{1}, v_{2}\right) \in\right] 1,3[\times R$.

Proof. - It is easy to see that for any fixed $(u, v) \in R \times R^{2}$ such that either $u \neq 0$ or $\left.v_{1} \notin\right] 1,3\left[\right.$ the integrand $L\left(u, v_{1}, v_{2}\right)$ is strictly convex as a function of $v=\left(v_{1}, v_{2}\right)$ at $v$.

Suppose that there exists a function $u \in W^{1,2}(\Omega)$ at which the functional does not have the weak-strong convergence property. By Theorem 1.5 we obtain that for any $x$ from a set $K \subset \Omega$ of nonzero measure $u(x)=0$, $\nabla u(x) \in[1,3] \times R$.

Using the theorem of almost everywhere differentiability of Sobolev functions (cf. [36, Th. 3.4.1]) we obtain that $\nabla u(x)=0$ for a.e. $x \in K$. This contradiction with the inclusion $\nabla u(x) \in[1,3] \times R$ completes the proof. 


\section{ACKNOWLEDGMENTS}

It is great pleasure to thank Prof. S. Müller for the help of different type. I am grateful also to G. Friesecke and P. Hajlasz for certain useful discussions, J. Kristensen for sending the monograph $[\mathrm{Kr}]$, and unknown referee for useful remarks and plenty helpful advices in English.

\section{REFERENCES}

[1] E. ACERB1 and N. FusCo, Semicontinuity problems in the calculus of variations, Arch. Rat. Mech. Anal, Vol. 86, 1984, pp. 125-145.

[2] G. AlberTI, A Lusin type theorem for gradients, J. Funct. Anal., Vol. 100, 1991, pp. $110-118$.

[3] E. J. BALDER, A general approach to lower semicontinuity and lower closure in optimal control theory, SIAM J. Control and Optimization, Vol. 22, 1984, pp. 570-598.

[4] J. M. BAL.J, Convexity conditions and existence theorems in nonlinear elasticity, Arch. Rat. Mech. Anal., Vol. 6, 1978, pp. 337-403.

[5] J. M. BALL, A version of the fundamental theorem for Young measures, in PDE's and Continutum Models of Phase Transirions, M. Rascle, D. Serre, M. Slemrod, eds, Lecture Notes in Physics 344, Springer-Verlag, 1989, pp. 207-215.

[6] J. M. BALL, and F, MURat, $W^{\prime / \mu}$-quasiconvexity and variational problems for multiple integrals, J. Funct. Anal., Vol. 58, 1984, pp. 225-253.

[7] J. M. BALL and K. ZHANG, Lower semicontinuity of multiple integrals and the biting lemma, Proc. Roy. Soc. Edinburgh, Sect A., Vol. 114. 1990, pp. 367-379.

[8] A. Cellina, On minima of functionals of gradient: necessary conditions, Nonlinear Analysis TMA, Vol. 20, 1993, pp. 337-341.

[9] A. CEllina, On minima of functionals of gradient: sufficient conditions, Nonlinear Analysis TMA, Vol. 20, 1993, pp. 343-347.

[10] $\wedge$. Cellifia and S. ZAGATTI, $\wedge$ version of Olech's lemma in a problem of the Calculus of Variations, SIAM J. Control and Optimization, Vol. 32, 1994, pp. 1114-1127.

111] B. Dacorogna, Weak continuity and weak lower semicontinuity of nonlinear problems, Lecture Notes in Math., Vol. 922, Springer-Verlag, 1982.

[12] B. Dacorogna, Direct methods in the Calculus of Variations, Springer-Verlag, 1989.

[13] I. EKELAND and R. TEMAM, Convex analysis and variational problems, Amsterdam, North-Holland, 1976.

[14] L. C. Evans and R. F. Gariepy, Some remarks on quasiconvexily and strong convergence, Proc. Roy. Soc: Edinburg, Sect. A, Vol. 106, 1987, pp. 53-61.

[15] G. FrIEsECKE, A necessary and sufficient condition for nonattainment and formation of microstructure almost everywhere in scalar variational problems, Proc. Rov. Soc: Edinburgh. Sect. A, Vol. 124, 1994, pp. 437-471.

[16] T. IWANIEC and C. SBORdONE, On the integrability of the jacobian under minimal hypotheses, Arch. Rat. Mech. Anal., Vol. 119, 1992, pp. 129-143.

[17] O. KaLAMAJSKA, Ural communication.

[18] D. Kinderlehrer and P. Pedregal, Characterization of Young measures generated by gradients, Arch. Rat. Mech. Anal.. Vol, 115, 1991. pp. 329-365.

[19] D. Kinderlehrer and P. Pedregal, Weak convergence of integrands and the Young measure representation, SIAM J. Math. Anal., Vol. 23, 1992, pp. 1-19.

[20] D. Kinderlehrer and P. Pedregal, Gradient Young measures generated by sequences in Sobolev spaces, J. Geon. Anal., Vol. 4, No. 1, 1994, pp. 59-90.

[21] J. KRISTENSEN, Finite functionals and Young measures generated by gradients of Sobolev functions, MAT-REPORT No. 1994-34, August 1994. 
[22] M. Krasnosel'SkI. and Y. RuTickiJ, Convex functions and Orlic: spaces, Groningen. Noordhoff, 1961.

[23] K. Kuratowski K and Rril-Nardzewski. A general theorem of selectors, Bull. Acad. Polon. Sci., Vol. XIII. No. 6, 1966, pp. 397-403.

[24] R. J. KNops and C. A. StuART, Quasiconvexity and Uniqueness of Equilibrium Solutions in Nonlinear Elasticity, Arch. Rat. Mech. Anal., Vol. 86, No. 3. 1984. pp. 233-249.

[25] P. Marcellini, Approximation of quasiconvex functions, and lower semicontinuity of multiple integrals. Manuscripta Math. Vol. 51, 1985, pp. 1-28.

[26] J. MaLY, Weak lower semicontinuity of polyconvex integrals, Proc. Ros. Sox Edimburgh. Sect A, Vol. 123. No. 4. 1993, pp. 681-691.

[27] C. B. Morrey, Multiple integrals in the Calculas of Variations, Springer-Verlag. 1960.

[28] P. Peidregal, Jensen's inequality in the calculus of variations, Differential and Integral Equations, Vol. 7, 1994, pp. 57-72.

[29] W. Rudin, Functional Analysis. Tata Mc Graw-Hill, 1985.

[30] M. SychEv. Necessary and sufficient conditions in theorems of lower semicontinuity and convergence with a functional. Russ. Acad. Sci. Sh. Math., Vol. 186. 1995, pp. 847-878.

[31] M. SYcHEV, Characterization of weak-strong convergence property of integral functionals by means of their integrands. Preprint 11, 1994. Inst. Math. Siberian Division of Russ. Acad. Sci., Novosibirsk.

[32] M. SYCHEV, A criterion for continuity of an integral functional on a sequence of functions, Siberian Math. J., Vol. 36. No. 1, 1995, pp. 146-156.

[33] A. VISINTIN, Strong convergence results related to strict convexity, Comm. Partial Differential Equations. Vol. 9. 1984, pp. 439-466.

[34] L. C. Young, Lectures on the Calculas of Variations and Optimal Control Theory, Saunders. 1969 (reprinted by Chelsea, 1980).

[35] L. C. Young, Generalized curves and the existence of an attained absolute minimum in the Calculus of Variations. Comptes Rendus de la Société des Sciencés et des Lettres de Varsovie, classe III, Vol. 30, 1937. pp. 212-234.

|36| W. P. Ziemer. Weakly differentiable fimctions, Springer-Verlag, New-York, 1989.

(Manuscript received March 13, 1996:

Ist revised version received June 18, 1996:

2d revised verwion September 24, 1996.) 